\title{
Evidence for wave harmonics in cool loops
}

\author{
E. O'Shea ${ }^{1}$, A. K. Srivastava ${ }^{1}$, J. G. Doyle ${ }^{1}$, and D. Banerjee ${ }^{2}$ \\ 1 Armagh Observatory, College Hill, Armagh BT61 9DG, N. Ireland, UK \\ e-mail: eos@arm.ac.uk \\ 2 Indian Institute of Astrophysics, II Block, Koramangala, Bangalore 560 034, India
}

Received 20 June 2007 / Accepted 12 August 2007

\section{ABSTRACT}

\begin{abstract}
Aims. Using temporal series image data from the Coronal Diagnostic Spectrometer (CDS) on SOHO, we study oscillations found in intensity (radiance) measurements from the transition region O V 629 line with the aim of finding evidence of wave harmonics in cool loops.

Methods. Using standard Fourier techniques, together with a pre-whitening method, we measure all statistically significant frequencies present in oscillations found in flaring active region loops at the temperature of $\mathrm{O} v$. By measuring the distances travelled by three Propagating Disturbances (PDs), estimates of their propagation speeds are calculated.

Results. Evidence is found for harmonics in the measured oscillations suggesting the presence of standing waves in the loops. We find that PDs in cool O V active region loops have sub-sonic velocities of between 12-24 $\mathrm{km} \mathrm{s}^{-1}$, suggesting they are moved either by slow mode (acoustic or slow magnetoacoustic) wave propagation or by bulk flows along the loops triggered by the flaring that also produces the PDs.

Conclusions. We find evidence that standing waves are present in flaring cool transition region loops. This evidence comes in the form of oscillations showing harmonic frequencies that match those expected for standing fast kink waves.
\end{abstract}

Key words. Sun: UV radiation - Sun: transition region - Sun: oscillations

\section{Introduction}

Nakariakov et al. (2004) and Tsiklauri et al. (2004) have previously suggested that oscillations with periods of $10-300 \mathrm{~s}$, frequently observed in flaring coronal loops, may be produced by the second standing harmonic of the acoustic mode. In a recent paper, De Moortel \& Brady (2007) found evidence of the 2nd and higher harmonics of flare-induced fast kink waves in coronal loops seen with TRACE. In this work, using time series of images obtained in the transition region (TR) line of O V $629 \AA$, we seek to find evidence for harmonics of standing waves in cool loops and to identify the particular wave mode involved.

\section{Observations and data reduction}

For these observations we used the $90^{\prime \prime} \times 240^{\prime \prime}$ wide slit of the Normal Incidence Spectrometer (NIS), part of the Coronal Diagnostic Spectrometer (CDS) on board the Solar and Heliospheric Observatory (SOHO) (Harrison et al. 1995).

The temporal series study AR_MOVIE was run during November 2005 in active region 10820 on the Western limb of the Sun. We shall discuss here two datasets from that observing run (see Table 1), concentrating on the TR line of O v $629.73 \AA$. The data were reduced using the latest versions of the standard CDS routines. For each of the 150 time frames in each dataset, the exposure time was $45 \mathrm{~s}$ leading to a cadence of $\approx 52 \mathrm{~s}$ and a Nyquist frequency of $\approx 9.6 \mathrm{mHz}$.

\section{Results}

It was noticed in examining dataset 33813 that a region at the southern end of the field-of-view (FOV) $\left(X \approx 975^{\prime \prime} \rightarrow 1010^{\prime \prime}\right.$
Table 1. AR_MOVIE/v1 time series datasets obtained using the $90^{\prime \prime} \times$ $240^{\prime \prime}$ CDS slit and an exposure time of $45 \mathrm{~s}$ in November 2005.

\begin{tabular}{llcl}
\hline \hline Date & Dataset & Pointing $(X, Y)$ & Start/End (UT) \\
\hline $10 / 11$ & 33813 & $1013,-254$ & $19: 46 / 21: 56$ \\
$11 / 11$ & 33821 & $1012,-255$ & $19: 36 / 21: 46$ \\
\hline
\end{tabular}

and $Y \approx-365^{\prime \prime} \rightarrow-340^{\prime \prime}$ ), cf. Fig. 1, possessed within it a Propagating Disturbance (PD) (Robbrecht et al. 2001). The PD does not propagate into this region but, instead, flares into life inside the loop it illuminates. It then begins to move along this loop from $X \approx 990^{\prime \prime}$ to $1005^{\prime \prime}$. At this location, it diminishes in intensity and disappears. Integrating along the curved trajectory of the maximum intensity in the PD gives a total distance travelled of $\approx 9144 \mathrm{~km}\left(7.6 \pm 1.9\right.$ pixels, 1 pixel $=1.68^{\prime \prime}$ and $\left.1^{\prime \prime}=715 \mathrm{~km}\right)$ in a time of $673 \mathrm{~s}$. That is, a velocity of $14 \pm 3.4 \mathrm{~km} \mathrm{~s}^{-1}$, which is below the sound speed at the temperature of $\mathrm{OV}\left(\approx 73 \mathrm{~km} \mathrm{~s}^{-1}\right)$. The PD is likely not moving perpendicular to the line-of-sight (LOS) and, therefore, this velocity should be taken as a lower bound value of the "true" velocity in the loop.

In Fig. 2a, we plot the variation of the maximum intensity found in the PD of Fig. 1 as a function of time, starting some 30 min before the start of the main flaring event (which occurs at $\approx 21: 31$ UT). As it is the variation of the maximum intensity, the position in the FOV is, therefore, not fixed. It can be seen from this plot that the intensity has a signature not dissimilar to that expected for a flare, that is, an initial sharp increase in intensity followed by a more gradual decrease with time.

Taking the oscillations found in the declining arm of the curve shown in Fig. 2a, i.e., between the times of 105-127 min, and removing the background intensity, produces the relative 


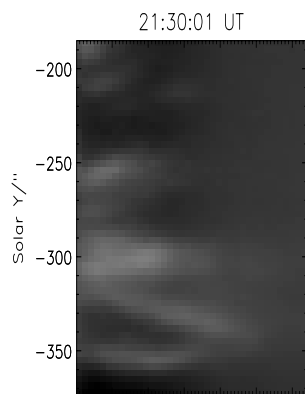

9809901000101010201030

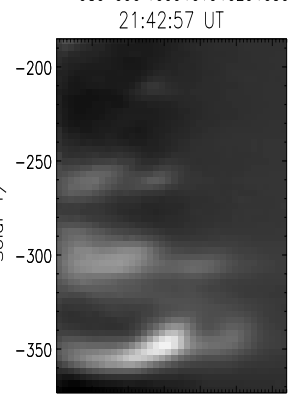

9809901000101010201030 Solor $X / "$

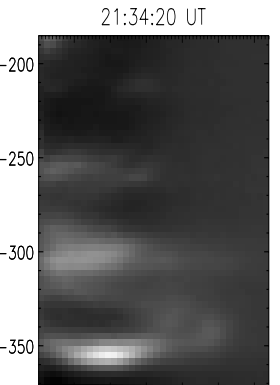

9809901000101010201030

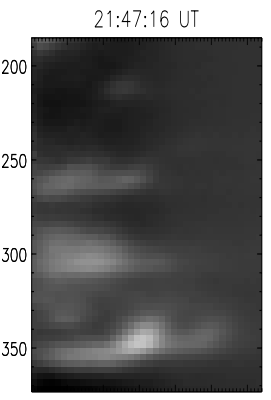

9809901000101010201030 Solor $X / "$
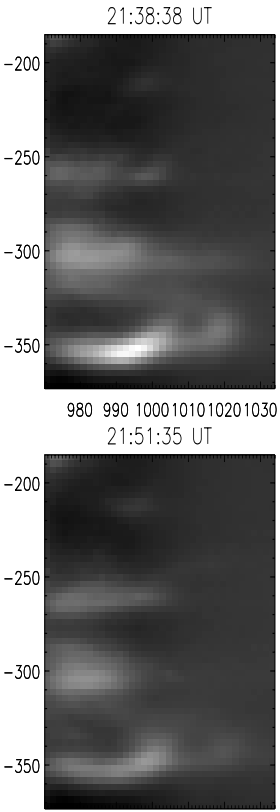
Solor X/"
9809901000101010201030

9809901000101010201030

Fig. 1. Evolution of the PD in dataset 33813 . The top $\approx 50^{\prime \prime}$ of the FOV has been omitted to remove the bright disk.
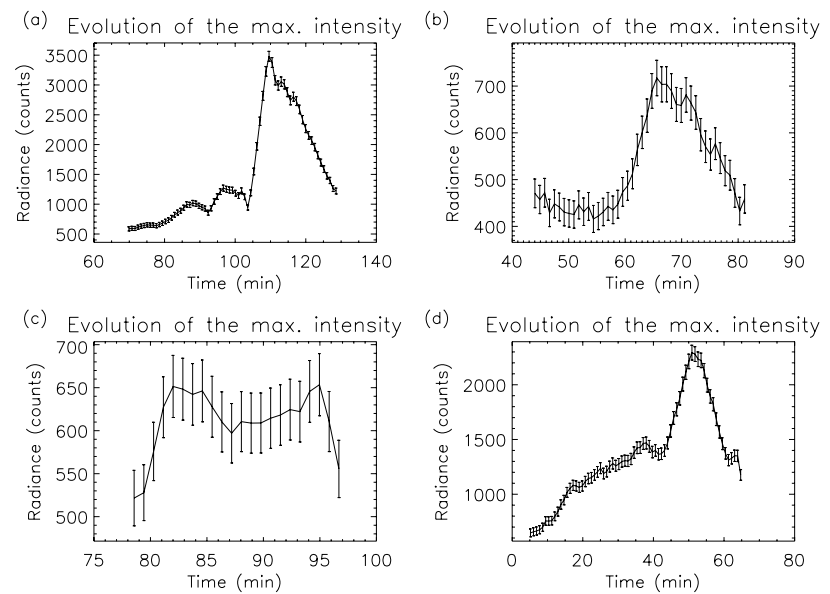

Fig. 2. Evolution of the maximum intensity in the a) PD of 33813 ; b) PD(a) of 33821r00; c) PD(b) of 33821; d) stationary flare of 33813. The time origins (or start times) are as given in Table 1.

intensity oscillations shown in Fig. 3a. Calculating the Fourier power spectrum (Fig. 3b), a frequency of $2.23 \mathrm{mHz}(448 \mathrm{~s}$ ) at the $96 \%$ confidence level, with an amplitude of $\approx 4 \%$, is found. The confidence level was estimated using a randomisation technique (O'Shea et al. 2001). Using a method of "pre-whitening", the first peak at $2.23 \mathrm{mHz}$ was suppressed to the level of noise (which we estimated from a randomisation of the whole time series data minus the component at $2.23 \mathrm{mHz}$ ). The result of this "pre-whitening" can be seen in Fig. $3 \mathrm{~b}$ where there is now a higher frequency oscillation of $4.46 \mathrm{mHz}$ present at a confidence level of $97 \%$ and a relative amplitude of $2.1 \%$. It will be noticed that this frequency is exactly twice that of the previous one, suggesting that we are seeing here harmonics of standing waves, e.g., 1 st and 2 nd, etc.

Examining dataset 33821, another PD, which we will call PD(a), was identified close to the solar limb (see Fig. 4). The
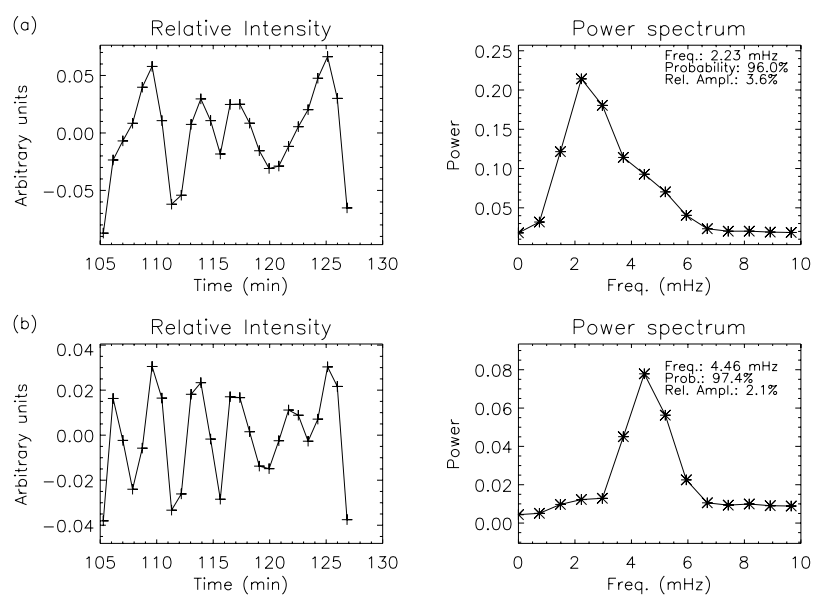

Fig. 3. a) Time series of the PD of 33813 and resulting power spectrum. b) Pre-whitened time series and resulting power spectrum. The time origin is as given in Table 1 for dataset 33813 .
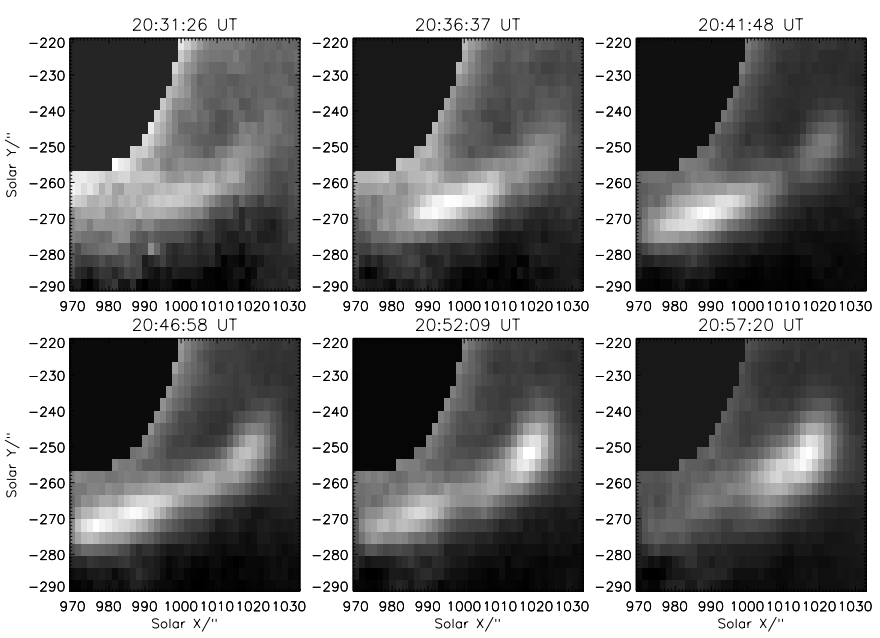

Fig. 4. Evolution with time of PD(a) of dataset 33821. The bright solar disk in the top left of the image has been blacked out.

flare-like evolution of the maximum intensity in the PD is shown in Fig. 2b. Integrating along the curved trajectory of the maximum intensity in the PD gives a total distance travelled of $\approx 23806 \mathrm{~km}(19.8 \pm 0.160$ pixels $)$ in a time of $1916 \mathrm{~s}$. That is, a sub-sonic velocity of $12.4 \pm 0.100 \mathrm{~km} \mathrm{~s}^{-1}$. In Fig. 5, we show the variation of the relative intensity in $\mathrm{PD}(\mathrm{a})$ between the observing times of $\approx 50-80 \mathrm{~min}$ (cf. Fig. 2). Oscillations at frequencies of $2.5 \mathrm{mHz}$ and $6.1 \mathrm{mHz}$ with amplitudes of $11.5 \%$ and $6.1 \%$, respectively, are found. Here the first and second frequencies are not in the expected 1:2 ratio. This may simply be a consequence of the crude frequency resolution of $\approx 0.5 \mathrm{mHz}$ resulting from the short duration $(\approx 30 \mathrm{~min})$ of the $\mathrm{PD} /$ flaring event.

In Fig. 6 we show a second PD, PD(b), occuring in the same loop system as $\mathrm{PD}(\mathrm{a})$. Integrating along the curved trajectory of the maximum intensity in the PD gives a total distance travelled of $\approx 25586 \mathrm{~km}(21.3 \pm 0.127$ pixels $)$ in a time of $1088 \mathrm{~s}$. That is, a sub-sonic velocity of $23.5 \pm 0.140 \mathrm{~km} \mathrm{~s}^{-1}$. The evolution of the maximum brightening in this PD (Fig. 2c) does not follow the shape expected for a flare-type event, having a largely flat profile with no rapid increase in intensity. Another difference is that this time series possesses just a single statistically significant frequency (>95\%) at $4.4 \mathrm{mHz}$. 

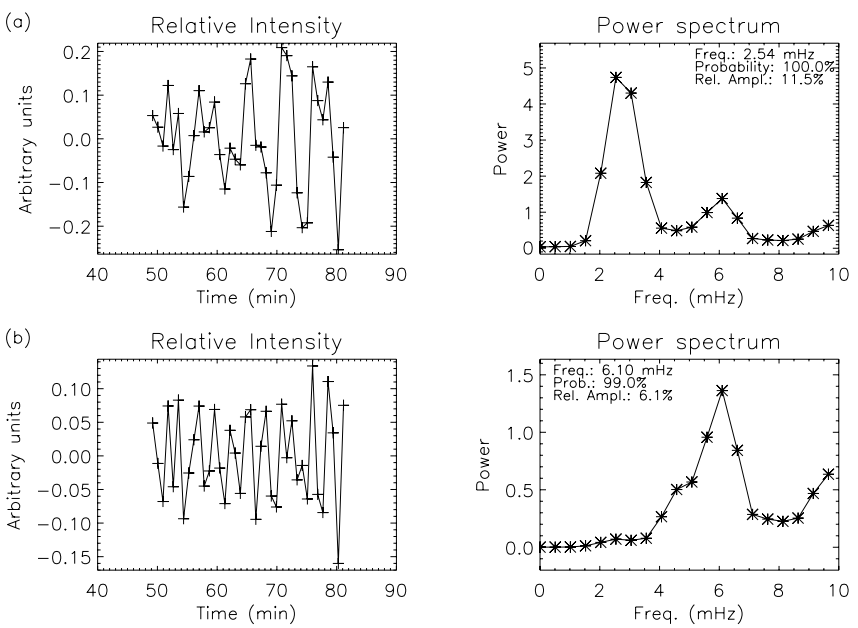

Fig. 5. a) Time series and power spectrum for $\operatorname{PD}(a)$ of 33821 . b) The pre-whitened time series and resulting power spectrum. The time origin is as given in Table 1 for dataset 33821 .
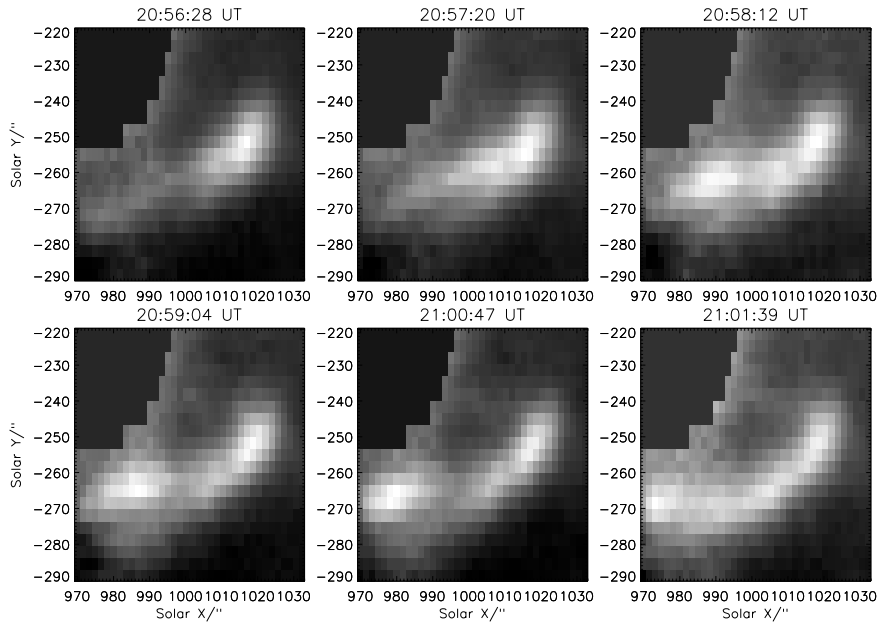

Fig. 6. Evolution with time of $\mathrm{PD}(\mathrm{b})$ of dataset 33821 . The bright solar disk in the top left of the image has been blacked out.

As a final example of flare-like events in cool O v loops, we show in Fig. 7 an example of a stationary flaring event observed in the 33813 dataset. Here the flare appeared at the apex of the loop $\left(X \approx 995^{\prime \prime} \rightarrow 1015^{\prime \prime}, Y \approx-340^{\prime \prime} \rightarrow-320^{\prime \prime}\right)$ and remained there for the duration of its existence, some $50 \mathrm{~min}$. The evolution of the maximum intensity in this stationary flare may be seen in Fig. 2d. In this time series, unlike, in Figs. $2 a$ and b, the measured oscillations are found in the rising arm of the light curve before the maximum brightening occurs (at $\approx 50 \mathrm{~min}$ ). This stationary flare lasting for over $50 \mathrm{~min}$ is similar to the longer flare type discussed in Fig. 1 of Nakariakov et al. (2004), where the oscillations also largely occur in the rising arm of the flare's light curve. This stationary flare possesses two harmonics of oscillation at $2.10 \mathrm{mHz}$ and $5.04 \mathrm{mHz}$ as shown in Fig. 8 . Given the frequency resolution of $\approx 0.4 \mathrm{mHz}$ in the $\approx 40 \mathrm{~min}$ of time series examined in Fig. 8, these two frequencies are likely to be harmonics of each other. We note the much larger amplitudes of oscillation (44\% and $26 \%$ ) in this stationary flare compared to the PDs of Figs. 3 and 5.

Examining Fig. 1, it can be seen that the essentially horizontal loop in which the PD is present extends out to a solar $X$ of $\approx 1010^{\prime \prime}$. At the pointing of the loop in $Y, Y \approx-350^{\prime \prime}$, the
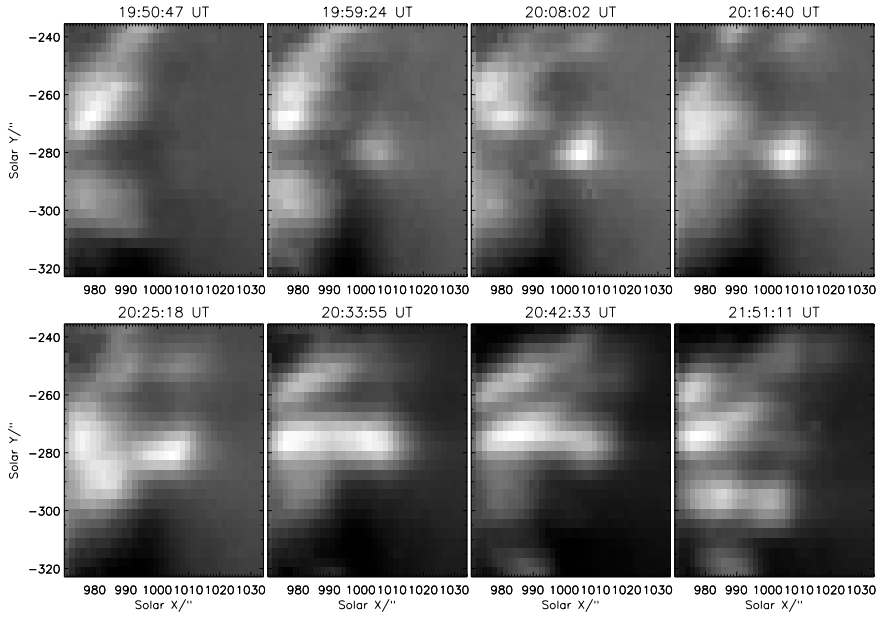

Fig. 7. Evolution with time of the stationary flare of dataset 33813.
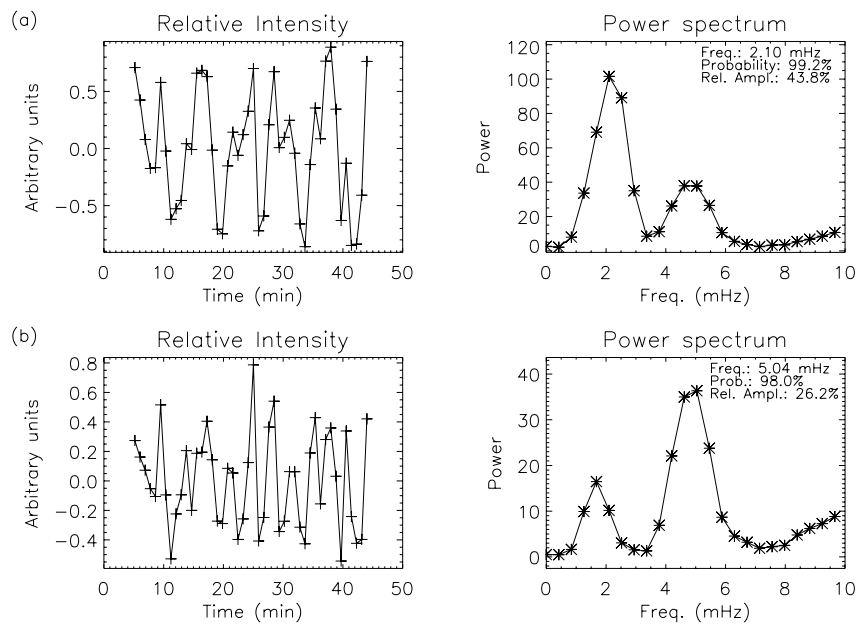

Fig. 8. a) Time series and power spectrum for the stationary flare of dataset 33813. b) The pre-whitened time series and power spectrum.

limb is located at a solar $X$ of $\approx 920^{\prime \prime}$. So the minimum distance from the loop apex to the footpoints is $\approx 90^{\prime \prime}$. If we assume the loop is semi-circular, and $90^{\prime \prime}$ is the radius, then the total loop length $(\mathrm{L})$ is $2.02 \times 10^{8} \mathrm{~m}$. Using this $\mathrm{L}$, and a temperature of $0.25 \mathrm{MK}$ for O v, in Eq. (3) of Tsiklauri et al. (2004) gives an expected 2nd harmonic period of $2.66 \times 10^{3} \mathrm{~s}(0.376 \mathrm{mHz})$ for standing acoustic waves. This is an order of magnitude larger than the "2nd" order harmonic found in Fig. 3, i.e., $224 \mathrm{~s}$ (or $4.46 \mathrm{mHz}$ ). This implies that, if the oscillations we see are due to low harmonic orders, then the propagation speed of the waves must also be larger than the sound speed by an order of magnitude. This then strongly suggests that we are observing fast kink waves, which are the only waves (cf. Aschwanden et al. 1999; Nakariakov \& Ofman 2001) that would match our observed periods of between 164-476 s (2.1-6.1 mHz). We note that intensity oscillations from fast kink waves can be observed despite their incompressibility as explained in Cooper et al. (2003). If we assume that the periods seen in Fig. 3 are due to fast kink waves, and that $2.23(\approx 448 \mathrm{~s}) \mathrm{mHz}$ is the 1 st order harmonic, then using Eq. (4) of Aschwanden et al. (1999), and our loop length (L) of $2.02 \times 10^{8} \mathrm{~m}$, gives a kink velocity, $\mathrm{c}_{k}$, of $901 \pm 325 \mathrm{~km} \mathrm{~s}^{-1}$. The large uncertainty comes from taking the frequency resolution of $0.773 \mathrm{mHz}$ in Fig. 3 as the (conservative) error in the measured frequency and assuming an error of $10 \%$ in the loop 

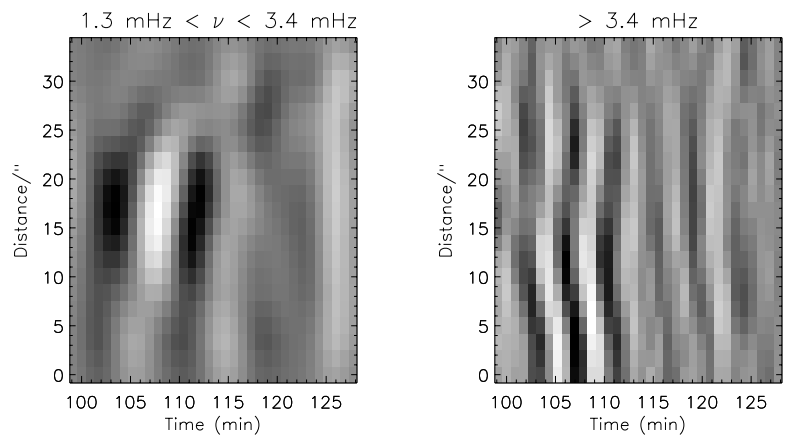

Fig. 9. $X-T$ diagram along the loop of dataset 33813 (cf. Fig. 1).

length (Nakariakov \& Ofman 2001). This kink velocity is close to values found in coronal loops, e.g., by Nakariakov \& Ofman (2001), once the uncertainties and the obvious differences in density between the TR and the corona are taken into account. This then suggests that the frequencies measured in Figs. 3, 5 and 8 are the 1 st and 2 nd order harmonics of fast kink waves. We show in Fig. 9 a space-time $(X-T)$ plot traced along the curved line of the loop illuminated in the bottom of Fig. 1, between $\left(X \approx 975^{\prime \prime} \rightarrow 1010^{\prime \prime}\right.$ and $\left.Y \approx-365^{\prime \prime} \rightarrow-340^{\prime \prime}\right)$, and at approximately the same times as in Fig. 3. In Fig. 9, we filtered the $X-T$, using standard Fourier hi- and band-pass filtering at each position along the $Y$ axis, in order to enhance those oscillations between $1.3-3.4 \mathrm{mHz}$ (the $1 \mathrm{st}$ harmonic) and those above $3.4 \mathrm{mHz}$ (the 2nd harmonic) (cf. Fig. 3). The presence of oscillations (the standing waves) at these frequencies is obvious, the light and dark stripes illustrating their peaks and troughs. $X-T$ plots of $\mathrm{PD}(\mathrm{a})$ and $\mathrm{PD}(\mathrm{b})$ produced similar results but we do not include them here due to a lack of space.

Movies of all the PDs shown here (Figs. 1, 4, 6 and 7) are available at http://star.arm.ac.uk/preprints.

\section{Discussion and conclusions}

In this letter we show the first evidence for harmonics of standing waves in cool loops, likely to be formed by the superposition of oppositely propagating fast kink waves. The standing waves are revealed as small flaring regions, either stationary or propagating at sub-sonic velocities of $12-24 \mathrm{~km} \mathrm{~s}^{-1}$ (the PDs), temporarily illuminate the loops to TR temperatures. The low velocities at which the PDs propagate suggests they are moved either by slow mode (acoustic or slow magnetoacoustic) wave propagation or by bulk plasma flows along the loops triggered by the same flaring that produces the PDs. The flaring activity may possibly also be the mechanism that is exciting the standing waves in the loops. Mendoza-Briceño \& Erdélyi (2006) found the presence of "cool gas blobs" (analagous to our PDs) travelling at mainly sub-sonic velocities to be a natural consequence of their model studying coronal loop oscillations triggered by random energy releases. They found, moreover, a suggestion that standing waves, propagating waves and moving "gas blobs" could all be present together, in excellent agreement with the results presented here. We note that Wang et al. (2005) found evidence of propagating "blobs" of plasma in hot loops as seen by SUMER/SOHO. Examining a number of our contemporaneously observed coronal lines (figures not included to save space), we find that there is no decrease or change in the coronal intensity relative to that seen in the cool loops, suggesting that the "blobs" seen in SUMER/SOHO hot loops may not be a direct high temperature counterpart of the PDs found here.

Acknowledgements. CDS is a part of SOHO, the Solar and Heliospheric Observatory, a project of international cooperation between ESA and NASA. This work was supported in part by a PRTLI research grant for Grid-enabled Computational Physics of Natural Phenomena (Cosmogrid) and PPARC grant PP/D001129/1. We thank the Royal Society and the British Council for funding visits between Armagh Observatory and the Indian Instutite of Astrophysics. We also thank the referee for comments and suggestions which helped to improve this paper.

\section{References}

Aschwanden, M. J., Fletcher, L., Schrijver, C. J., \& Alexander, D. 1999, ApJ, 520,880

Cooper, F. C., Nakariakov, V. M., \& Tsiklauri, D. 2003, A\&A, 397, 765

De Moortel, I., \& Brady, C. S. 2007, ApJ, 664, 1210

Harrison, R. A., Sawyer, E. C., Carter, M. K., et al. 1995, Sol. Phys., 162, 233

Mendoza-Briceño, C. A., \& Erdélyi, R. 2006, ApJ, 648, 722

Nakariakov, V. M., \& Ofman, L. 2001, A\&A, 372, L53

Nakariakov, V. M., Tsiklauri, D., Kelly, A., et al. 2004, A\&A, 414, L25

O'Shea, E., Banerjee, D., Doyle, J. G., Fleck, B., \& Mugtagh, F. 2001, A\&A, 368,1095

Robbrecht, E., Verwichte, E., Berghmans, et al. 2001, A\&A, 370, 591

Tsiklauri, D., Nakariakov, V. M., Arber, T. D., \& Aschwanden, M. J. 2004, A\&A, 422, 351

Wang, T. J., Solanki, S. K., Innes, D. E., \& Curdt, W. 2005, A\&A, 435, 753 